\title{
CARACTERIZAÇÃO ALIMENTAR DE CRIANÇAS COM CARDIOPATIAS CONGÊNITAS ${ }^{1}$
}

\author{
FOOD CHARACTERISTICS OF CHILDREN WITH \\ CONGENITAL HEART DISEASE
}

\section{CARACTERIZACIÓN ALIMENTARIA DE LOS NIÑOS CON CARDIOPATÍAS CONGÉNITAS}

\author{
Flávia Paula Magalhães Monteiro* \\ Mabelly Barbosa Lopes Ramos* \\ Ticiana de Oliveira Pontes ${ }^{* *}$ \\ Gabrielle FÁvaro Holanda ${ }^{* * * *}$ \\ Huana Carolina CÂndido Morais ${ }^{* * * *}$ \\ Thelma Leite De Araujo
}

\begin{abstract}
RESUMO
Caracterizar dados sociodemográficos e história alimentar das crianças portadoras de cardiopatias congênitas com até 24 meses de vida. Estudo transversal e observacional desenvolvido em duas instituições de saúde: ambulatorial e hospitalar. Coleta de dados em janeiro a junho de 2009, utilizando formulário contendo dados sociodemográficos, dados relativos à situação de saúde-doença e dados da história alimentar das crianças. Respeitaram-se os aspectos éticos. Avaliaram-se 132 crianças predominantemente de Fortaleza-CE, sexo masculino, mediana até 8,5 meses de vida. A história alimentar evidenciou uso de leite industrializado e complementos alimentares (49,6\%), leite materno (11,9\%) e leite materno com leite industrializado (7,7\%). Problemas durante alimentação: náuseas, vômitos, refluxo gastroesofágico, tosse, dispnéia e cianose. Segundo as mães, as crianças apresentavam apetite preservado $(74,2 \%)$ e sem dificuldades de mastigação, sucção e deglutição (93,8\%). Para minimizar o problema da desnutrição dessas crianças, os enfermeiros podem atuar de forma decisiva mediante o ajuste de medidas alimentares da criança consoante adequação do hábito alimentar.
\end{abstract}

Palavras chave: Criança, cardiopatias congênitas, comportamento alimentar, enfermagem.

\footnotetext{
ABSTRACT

Objective: To characterize the demographics and dietary history of children up to 24 months old with congenital heart disease. Methodology: Cross-sectional observational study carried out in a Hospital and Ambulatory care setting. Data Collection was conducted from January to June 2009 using a form that includes the socio

${ }^{1}$ Trabalho extraído de parte da dissertação de Mestrado apresentada ao Programa de Pós-Graduação em Enfermagem da Universidade Federal do Ceará/UFC, com apoio financeiro do CNPq.

* Enfermeira. Membro do Projeto Cuidado em Saúde Cardiovascular. Bolsista CNPq processo No 141765/2010-0. Email: flaviapmm@yahoo.com.br

${ }^{* *}$ Enfermeira. Email: bellybbs@yahoo.com.br

${ }^{* * *}$ Enfermeira. Email: ticiop@hotmail.com

${ }^{* * * *}$ Enfermeira. Membros do Projeto Cuidado em Saúde Cardiovascular. Email: gabyfavaro@yahoo.com.br

${ }^{* * * * *}$ Enfermeira. Membros do Projeto Cuidado em Saúde Cardiovascular. Email: huanacarolina@yahoo.com.br

${ }_{* * * * * *}^{*}$ Enfermeira. Professora associada da Universidade Federal do Ceará (UFC). Coordenadora do Projeto Cuidado em Saúde Cardiovascular. Email: thelmaaraujo2003@yahoo.com.br
} 
demographic characteristics, health and disease status and feeding history of children, as well. Ethical aspects were observed. Results: From the 132 children evaluated, most of them were predominantly from Fortaleza, male, and median age of 8.5 months. The feeding history revealed intakes of industrialized milk and food supplements (49.6\%), breastfeeding (11.9\%), and breastfeeding along with industrialized milk (7.7\%). Problems during feeding: nausea, vomiting, gastroesophageal reflux, cough, dyspnoea and cyanosis. According to the mothers, children had preserved appetite $(74.2 \%)$ and no difficulty for chewing, sucking and swallowing (93.8\%). Conclusion: In order to minimize malnutrition problem in these children, nurses can act decisively by adjusting diet measures depending on the adequacy of child feeding practices.

Key words: Child, heart defects, congenital, feeding behavior, nursing.

\section{RESUMEN}

Objetivo: Caracterizar condiciones sociodemograficas y la historia de la alimentación de los niños con cardiopatías congénitas hasta los 24 meses de vida. Material y método: Estudio transversal y observacional desarrollado en dos instituciones de salud: ambulatoria y hospitalaria. Recolección de datos desde enero a junio de 2009 utilizando un formulario que recoge las características sociodemográficas, datos sobre la situación de salud y enfermedad y acerca de la historia alimentaria de los niños Los aspectos éticos fueron respetados. Se evaluaron 132 niños predominantemente de Fortaleza-CE, varones, con mediana hasta 8,5 meses de vida. La historia alimentaria reveló el uso de leche industrializada y complementos alimenticios $(49,6 \%)$, leche materna $(11,9 \%)$ y la leche materna con leche industrializada $(7,7 \%)$. Problemas durante la alimentación: náuseas, vómitos, reflujo gastroesofágico, tos, disnea y cianosis. De acuerdo con las madres, los niños presentaban apetito conservado $(74,2 \%)$ y sin dificultad para masticación, succión y deglutición (93,8\%). Para minimizar el problema de la desnutrición de estos niños, los enfermeros pueden actuar de manera decisiva mediante el ajuste de las medidas alimentarias de niños en función de la adecuación del hábito alimentario.

Palabras clave: Niño, cardiopatías congénitas, conducta alimentaria, enfermería.

Fecha recepción: 23/11/10 Fecha aceptación: 12/04/12

\section{INTRODUÇÃO}

As cardiopatias congênitas representam anomalias cuja expressão clínica inclui defeitos estruturais e funcionais que atingem $9 \mathrm{em}$ cada 1.000 crianças nascidas na população americana. Diante deste fato, cerca de 1,3 milhão de americanos com defeitos cardiovasculares estão vivos hoje e pelo menos 36.000 crianças nascem com estes defeitos todo ano (1).

Em um estudo colombiano foi encontrada uma prevalência de 1,2 casos de nascimentos por 1.000 nascidos vivos com cardiopatia congênita entre os anos 2001 e 2005 , sendo que $65,5 \%$ tinham cardiopatias graves (2). No geral, essas crianças proveem de famílias com baixo poder aquisitivo e baixa escolaridade, o que dificulta o acesso da criança à instituição de saúde, bem como na identificação precoce de sinais/sintomas peculiares da doença pela mãe ou responsáveis da criança. Especificamente, no presente estudo, as crianças e seus familiares apresentavam dificuldade de acesso ao serviço de saúde: dependiam de transportes disponibilizados pelo município ao qual pertenciam.

Somando-se a isso, os familiares, muitas vezes, recorriam ao auxílio-salário fornecido pelo Governo Federal, tendo em vista que as crianças passavam a ser cadastradas como portadoras de doenças crônicas que tinham direito ao apoio financeiro para complemen- 
tar despesas alimentares e outros de caráter educacional.

Com base nisso, as crianças portadoras de cardiopatias congênitas comumente apresentam além de hipóxia, hipermetabolismo, dificuldades alimentares, problemas de sucção, diminuição de apetite, os quais originam desequilíbrios nutricionais. Somandose a isso, a má absorção presente nas crianças cardiopatas limita significativamente a tolerância alimentar e afeta a capacidade da criança para maximizar o consumo calórico, bem como diminui a absorção de nutrientes (3). Dessa forma, a desnutrição é constante nessas crianças, independente da natureza do defeito cardíaco e da presença ou não de cianose (4).

Para os familiares, o momento das refeições é geralmente tenso e estressante, visto que as crianças frequentemente recusam os alimentos (5). Provavelmente, esse problema decorre de outros fatores os quais incluem diminuição da capacidade gástrica causada por hepatomegalia e o retardo no esvaziamento gástrico secundário ao baixo débito cardíaco. Além disso, as infecções respiratórias frequentes podem reduzir a função do músculo esquelético e aumentar o risco de pneumonias no pós-operatório bem como afetar a habilidade da criança na coordenação da sucção-deglutição-respiração para o êxito da alimentação oral $(3,6)$.

Diante das dificuldades mencionadas na alimentação, outras manifestações clínicas estão presentes nas cardiopatias congênitas, tais como: distúrbios esofágicos, incluindo refluxo gastroesofágico (RGE), malformações anorretais, atresia duodenal e onfalocele associadas à tetralogia de fallot e ao defeito do septo ventricular, as quais podem interferir diretamente no equilíbrio nutricional da criança e atrasar a intervenção cirúrgica bem como aumentar o risco para complicações pós-operatórias (6).

Em face disso, para minimizar os problemas durante a alimentação das crianças nascidas cardiopatas, os enfermeiros podem atuar de forma decisiva. Eles têm papel significativo no ajuste de medidas alimentares da criança em consonância com a adequação do hábito alimentar mediante estratégias nutricionais, com o intuito de prevenir possíveis complicações nos períodos intra e pós-operatórios.

Dessa forma, esse estudo tem o intuito de caracterizar os aspectos sociodemográficos, situação de saúde-doença e a história alimentar das crianças com cardiopatias congênitas com até 24 meses de vida, com o intuito de subsidiar as propostas de intervenção na assistência de enfermagem.

\section{MATERIAL E MÉTODO}

Estudo transversal com enfoque quantitativo realizado no período de janeiro a junho de 2009 com 132 crianças portadoras de cardiopatias congênitas com até 24 meses de vida, recrutadas aleatoriamente por amostra de conveniência em duas instituições de saúde com atendimento ambulatorial e hospitalar, especializadas em doenças cardíacas de Fortaleza, capital do Estado do Ceará/Brasil.

A amostra por conveniência caracteriza-se por alocar sujeitos por métodos não-aleatórios, que atendem aos critérios de inclusão e são de fácil acesso ao investigador. Dessa forma, à medida que adentrava à instituição, a criança era avaliada pelo pesquisador quanto aos critérios de inclusão previamente estabelecidos. Por conseguinte, a seleção de 132 crianças foi obtida por um período de seis meses.

Vale ressaltar que a inclusão de duas instituições de saúde com atendimento diferenciado justificou-se pela necessidade de se ampliar a amostra de crianças, na tentativa de torná-la representativa e suficiente para implementar testes estatísticos específicos. Esse fato pode ser considerado também uma limitação do estudo, tendo em vista que as crianças agrupadas apresentam característi- 
cas clínicas evidentes.

A instituição hospitalar é centro de referência em doenças cardiopulmonares e atende uma clientela advinda de todo o Estado do Ceará. Nela, a unidade de internação pediátrica foi lócus deste estudo. Esta unidade caracteriza-se por receber crianças para acompanhamento clínico, definição diagnóstica e tratamento cirúrgico corretivo ou paliativo de cardiopatias.

A outra instituição, de caráter ambulatorial, atende crianças e adolescentes na prevenção, diagnóstico e tratamento de patologias cardíacas por meio de consultas ambulatoriais, reabilitação pós-operatória e exames cardiográficos. Ressalta-se que essa última instituição tem caráter filantrópico e recebe diariamente mães e crianças desprovidas de recursos financeiros de diferentes localidades circunvizinhas à sede do município onde foi realizado o estudo. A instituição conta com uma casa adstrita ao ambulatório para acomodar pacientes e familiares, os quais recebem alimentação e apoio de uma equipe multiprofissional.

A coleta dos dados teve início em janeiro de 2009 e foi concluída em março do mesmo ano nas duas instituições de saúde. Foi realizada por meio de entrevista, com aplicação de formulário estruturado em questões abertas e fechadas subdividido em: dados sociodemográficos, dados relativos à situação de saúde-doença e dados da história alimentar das crianças. Os dados sociodemográficos apresentaram variáveis relacionadas ao sexo, idade, procedência e naturalidade, renda familiar, escolaridade materna, posição da criança na família; os relativos à situação de saúde-doença incluíram diagnóstico médico, tipo de cardiopatia, queixa principal, realização de cirurgias e co-morbidades presentes e informações familiares sobre cardiopatias. E, finalmente, os dados alimentares abordaram variáveis: tipo de alimentação, intercorrências durante a amamentação e/ou refeições, dificuldades de mastigação/sucção/deglutição.
Inicialmente, foram coletados dados primários mediante entrevista com os pais da criança. Em seguida, dados secundários foram coletados por consultas ao prontuário ou fichas ambulatoriais com o intuito de complementar as informações fornecidas pelos pais. Destaca-se que a maior parte das entrevistas foi realizada com a mãe da criança, algumas entrevistas foram feitas com o pai e, também poucas, incluíram ambos os pais ou mesmo outros familiares como avós. Para o estudo, optou-se por denominar o informante de pais.

Para a elaboração do formulário, foi realizado um teste piloto com seis crianças, provenientes das duas instituições de saúde, porém essas crianças não foram incluídas na amostra do estudo. Esse procedimento ocorreu para avaliar a adequabilidade das variáveis propostas na investigação, bem como a receptividade de pais e das crianças no estudo.

Os dados coletados foram compilados em planilhas do Excel e no software SPSS versão 13.0. Para a análise descritiva consideraram-se as frequências absolutas e percentuais. Realizou-se a análise dos dados numéricos por meio dos testes estatísticos: Kolmogorov-Smirnov, para verificação de normalidade dos dados numéricos, e o teste de Levene, para verificação de homogeneidade das variâncias (homocedasticidade).

Finalmente, os dados foram expostos em tabelas, com medidas de tendência central e percentis.

O projeto, como parte de um estudo de dissertação, foi encaminhado ao Comitê de Ética em Pesquisa do Hospital lócus do estudo e aprovado em protocolo número 569/08, com o intuito de atender às exigências da resolução 196/96 sobre pesquisa com seres humanos do Conselho Nacional de Saúde/ Ministério da Saúde (7).

Para isso, os pais das crianças participantes do estudo foram esclarecidos tanto sobre o objetivo deste como sobre o sigilo quanto às informações e identidades e foram solicitados a dar a anuência ao estudo mediante a 
assinatura do termo de consentimento livre e esclarecido.

\section{RESULTADOS}

No estudo, avaliaram-se 132 crianças com até 24 meses, portadoras de cardiopatias congênitas atendidas nos locais supracitados. Com a finalidade de caracterizar os dados socio- demográficos (renda familiar, escolaridade materna, procedência, naturalidade, ocupação materna, número de dependentes da renda, estado civil da mãe), as crianças foram distribuídas quanto ao sexo, procedência e naturalidade, instituição de saúde, condição clínica de saúde, presença de co-morbidades e cardiopatias entre os familiares, posição do filho na família, estado civil, escolaridade, situação ocupacional da mãe, renda familiar e número de dependentes (Tabela 1).

Tabela 1. Distribuição das crianças portadoras de cardiopatias congênitas segundo as características sociodemográficas, situação saúde-doença, condições clínicas e familiares. Fortaleza, 2009.

\begin{tabular}{|c|c|c|}
\hline Variáveis & $\mathbf{N}$ & $\%$ \\
\hline \multicolumn{3}{|l|}{ 1. Sexo } \\
\hline Masculino & 68 & 51,5 \\
\hline$\underline{\text { Feminino }}$ & 64 & 48,5 \\
\hline Total & 132 & 100,0 \\
\hline \multicolumn{3}{|l|}{ 2. Procedência } \\
\hline Capital & 78 & 59,5 \\
\hline Interior & 51 & 39 \\
\hline Outro Estado & 2 & 1,5 \\
\hline Total & 131 & 100,0 \\
\hline \multicolumn{3}{|l|}{ 3. Naturalidade } \\
\hline Capital & 82 & 62,6 \\
\hline Interior & 44 & 33,6 \\
\hline Outro Estado & 5 & 3,8 \\
\hline Total & 131 & 100,0 \\
\hline \multicolumn{3}{|c|}{ 4. Instituição de saúde } \\
\hline Hospitalar & 31 & 23,5 \\
\hline Ambulatorial & 101 & 76,5 \\
\hline Total & 132 & 100,0 \\
\hline \multicolumn{3}{|c|}{ 5. Condição clínica } \\
\hline Pré-operatória & 111 & 84,1 \\
\hline Pós-operatória & 21 & 15,9 \\
\hline Total & 132 & 100,0 \\
\hline \multicolumn{3}{|c|}{ 6. Co-morbidades } \\
\hline Presente & 37 & 28,2 \\
\hline Ausente & 94 & 71,8 \\
\hline Total & 131 & 100,0 \\
\hline \multicolumn{3}{|c|}{ 7. Familiares cardiopatas } \\
\hline Presente & 58 & 44,3 \\
\hline Ausente & 73 & 55,7 \\
\hline Total & 131 & 100,0 \\
\hline
\end{tabular}


Continuação Tabela 1.

\begin{tabular}{|c|c|c|c|c|c|c|}
\hline \multicolumn{7}{|l|}{ 8. Estado civil da mãe } \\
\hline Com companheiro & \multicolumn{3}{|c|}{106} & \multicolumn{3}{|c|}{80,9} \\
\hline$\underline{\text { Sem companheiro }}$ & \multicolumn{3}{|c|}{25} & \multicolumn{3}{|c|}{19,1} \\
\hline Total & \multicolumn{3}{|c|}{131} & \multicolumn{3}{|c|}{100,0} \\
\hline \multicolumn{7}{|l|}{ 9. Ocupação materna } \\
\hline Cuidadoras do lar & \multicolumn{3}{|c|}{88} & \multicolumn{3}{|c|}{69,8} \\
\hline Outros & \multicolumn{3}{|c|}{38} & \multicolumn{3}{|c|}{30,2} \\
\hline \multirow[t]{3}{*}{ Total } & \multicolumn{3}{|c|}{126} & \multicolumn{3}{|c|}{100,0} \\
\hline & \multirow{2}{*}{ Média } & \multirow{2}{*}{$\mathrm{DP}$} & \multicolumn{3}{|c|}{ Percentis } & \multirow[t]{2}{*}{$\mathrm{K}-\mathrm{S}$ (sig) } \\
\hline & & & $\mathrm{P}_{25}$ & $\mathrm{P}_{50}$ & $\mathrm{P}_{75}$ & \\
\hline 10. Idade da criança (meses) & 9,43 & 6,08 & 4,00 & 8,5 & 15,00 & 0,008 \\
\hline 11. Renda familiar ${ }^{*}$ & 1,54 & 0,92 & 1,00 & 1,00 & 2,00 & 0,000 \\
\hline 12. Número de dependentes da renda & 4,24 & 1,40 & 3,00 & 4,00 & 5,00 & 0,000 \\
\hline 13. Escolaridade materna (anos) & 9,09 & 3,17 & 7,00 & 9,00 & 12,00 & 0,001 \\
\hline 14. Posição do filho (ordem) ${ }^{* *}$ & 2,04 & 1,41 & 1,00 & 2,00 & 3,00 & 0,000 \\
\hline
\end{tabular}

*Renda familiar em salários mínimos, valor: U\$ 890,12; * Posição do filho portador de cardiopatias congênitas na família; DP: Desvio Padrão; $\mathrm{P}_{25}$ : Percentil 25; $\mathrm{P}_{50}$ : Percentil 50 ou mediana; $\mathrm{P}_{75}$ : Percentil 75; K-S (sig): Teste de Kolmogorov-Smirnov.

Conforme se observa, pelos dados da Tabela 1, houve discreta predominância do sexo masculino $(51,5 \%)$. No referente à procedência e à naturalidade, as crianças, em sua maioria, eram naturais da capital do Estado do Ceará (62,6\%) e dela procediam (59,5\%).

Quanto à instituição de saúde, a maior parte das crianças recebia atendimento ambulatorial $(76,5 \%)$, sob condição pré-operatória $(84,1 \%)$ e sem relatos de co-morbidades $(71,8 \%)$. No entanto, em algumas crianças $(28,2 \%)$ foi possível identificar co-morbidades relacionadas a infecções respiratórias de repetição, hérnias, síndromes genéticas (síndrome de Down) e anemias. Tratando-se da presença de cardiopatias entre os familiares, quase metade das crianças $(44,3 \%)$ tinha algum parente com cardiopatia.

Mesmo em número reduzido, 15,9\% das crianças se encontravam em condição pósoperatória de cirurgias paliativas e reparadoras. Contudo, o tempo de pós-operatório se concentrou entre um e treze meses, considerando o critério de inclusão de um mês após o procedimento cirúrgico.
No referente às características das mães, a maioria delas vivia com companheiro $(80,9 \%)$ e se ocupava nas atividades do lar $(69,8 \%)$. Além disso, diferentes tipos de ocupação foram identificadas $(30,2 \%)$, entre eles: professoras, domésticas, costureiras, agricultoras, vendedoras, comerciantes autônomas, diaristas, cozinheiras, auxiliares administrativas e estudantes.

As variáveis idade da criança (meses), renda familiar, número de dependentes da renda, escolaridade materna (em anos) e a posição do filho na família (em ordem) evidenciaram distribuição assimétrica pelo teste de Kolmogorov-Smirnov $(\mathrm{p}<0,05)$ e foram analisadas quanto às medianas.

Em face disso, 50\% das crianças apresentaram idade até 8,50 meses e seus familiares informaram renda mensal de até um salário mínimo e até quatro pessoas como dependentes desse salário. Em relação ao tempo de escolaridade, metade das mães informou ter até nove anos de estudo. Sobre a ordem na família, cerca de $50 \%$ das crianças avaliadas ocupavam até a segunda posição. 
Vale ressaltar que as variáveis procedência e naturalidade, co-morbidades, familiares cardiopatas, estado civil e ocupação das mães não corresponderam à totalidade da amostra deste estudo, pois, por problemas técnicoadministrativos, alguns dados não foram coletados no momento da entrevista.
A seguir, observa-se a distribuição das crianças portadoras de cardiopatias congênitas quanto à história de alimentação, englobando o tipo de alimentação, intercorrências durante a alimentação e amamentação, apetite da criança e dificuldades alimentares (Tabela 2).

Tabela 2. Distribuição das crianças portadoras de cardiopatias congênitas quanto à história de alimentação. Fortaleza, 2009.

\begin{tabular}{lrr}
\hline Variáveis & $\mathrm{N}$ & $\%$ \\
\hline 1. Tipo de alimentação & & \\
Leite industrializado + complementos alimentares & 61 & 49,6 \\
Leite materno + complementos alimentares & 27 & 20,8 \\
Leite materno exclusivo & 21 & 11,9 \\
Leite industrializado & 13 & 10,0 \\
Leite materno + leite industrializado & 10 & 7,7 \\
\hline Total & 132 & 100,0 \\
\hline 2. Intercorrências durante amamentação & & \\
Não & 28 & 52,8 \\
Sim & 25 & 47,2 \\
\hline Total & 53 & 100,0 \\
\hline 3. Apetite & & \\
Preservado & 98 & 74,2 \\
Diminuído & 26 & 19,7 \\
Aumentado & 8 & 6,1 \\
\hline Total & 132 & 100,0 \\
\hline 4. Intercorrências durante refeições & & \\
Não & 102 & 77,3 \\
Sim & 30 & 22,7 \\
\hline Total & 132 & 100,0 \\
\hline 5. Dificuldade de mastigação/sucção/deglutição & \multicolumn{2}{c}{} \\
Não & 120 & 93,8 \\
Sim & 8 & 6,2 \\
\hline Total & 128 & 100,0 \\
\hline
\end{tabular}

Considerando a história de alimentação das crianças portadoras de cardiopatias congênitas com até 24 meses de vida, os dados evidenciam que predominou o uso de leite industrializado e complementos alimentares $(49,6 \%)$, caracterizados por sucos, frutas amassadas e sopas de legumes. Outra parcela de crianças $(11,9 \%)$ recebia leite materno exclusivo e outras recebiam leite materno complementado com leite industrializado $(7,7 \%)$. É importante destacar que das 132 crianças avaliadas, apenas 19 delas estavam em aleitamento materno exclusivo.

Ao se avaliar os principais problemas durante a alimentação, consoante observou-se, a maioria das crianças não apresentou intercorrências durante as refeições (77,3\%) e amamentação (52,8\%), respectivamente. Entre os problemas manifestados durante a alimentação pelas demais, destacaram-se: náuseas, vômitos, refluxo gastroesofágico, tosse, dispnéia e cianose. Além disso, segun- 
do a percepção dos pais, um número expressivo de crianças apresentava apetite preservado $(74,2 \%)$, e sem dificuldades relacionadas à mastigação, sucção e deglutição (93,8\%).

Cabe salientar que as variáveis: intercorrências durante a amamentação e dificuldade de mastigação/sucção/deglutição, não corresponderam à totalidade da amostra deste estudo, pois, no instrumento utilizado, quando a criança não se encontrava em amamentação, o avaliador suprimia as perguntas referentes às variáveis.

\section{DISCUSSÃO E COMENTÁRIO}

Em virtude da doença, as crianças portadoras de cardiopatias congênitas requerem acompanhamento especializado desde o nascimento, por meio de estratégias de cuidado que exigem do enfermeiro o reconhecimento sobre as particularidades apresentadas pela doença e, sobretudo, em relação à condição alimentar dessas crianças.

Somando-se a isso, diferentes fatores podem contribuir para o desenvolvimento de desequilíbrios nutricionais nas crianças portadoras de cardiopatias congênitas. Entre eles estão: o tipo e magnitude da doença cardíaca, distúrbios no metabolismo energético caracterizado pelo aumento do gasto energético, dificuldades alimentares decorrentes da saciedade precoce, anorexia e alterações na função gastrointestinal, incluindo dificuldades no aproveitamento do alimento para o crescimento (8).

No concernente à história alimentar das crianças cardiopatas no presente estudo, a maioria delas fazia uso de leite industrializado complementado com outros alimentos, introduzidos em períodos regulares do dia, no total de quatro a nove refeições diárias. Ainda como observado, o período de transição para outras fontes de nutrientes começou por volta do segundo mês de vida da criança. Cabe uma ressalva: por dificul- dades de avaliar o quantitativo alimentar, esse estudo se restringiu apenas à avaliação qualitativa da alimentação infantil. Este tipo de levantamento provê o profissional do conhecimento sobre todo o processo alimentar, envolvendo aspectos relacionados à cultura, religião, hábitos, além de características da sociedade na qual a criança e seus familiares estão inseridos (9).

Quando se abordam os diferentes aspectos referentes à alimentação da criança com malformações cardíacas congênitas, é importante considerar que desnutrição e cardiopatias congênitas estão fortemente relacionadas. Nessa ordem, algumas limitações são reconhecidas e evidenciadas pela literatura, tais como problemas respiratórios reincidentes, hipóxia e constantes internações hospitalares, as quais contribuem diretamente para o aparecimento de alterações no peso e no comprimento infantil (10).

Dessa forma, a alimentação das crianças portadoras de cardiopatias congênitas é assunto de extrema relevância e deve receber atenção específica dos profissionais da saúde que atuam diretamente no cuidado a essa clientela. Assim, ao nascer, o leite materno é o alimento mais apropriado, sendo indicado por suas propriedades imunológicas e nutricionais de alto valor biológico, independente da condição de saúde apresentada pelo bebê.

$\mathrm{O}$ aleitamento materno é ideal para fornecer o crescimento e desenvolvimento adequado da criança nos primeiros seis meses de vida, devendo ser mantido pela mãe ao longo dos doze meses e, por volta do quarto ou sexto mês outras fontes de nutrientes devem ser acrescentadas à alimentação (1).

No estudo, parte das crianças fazia uso de leite materno exclusivo e os motivos que as levaram ao desmame foram dificuldades de sucção pela dispnéia, cianose, hospitalização e lesões mamilares. Em decorrência da dispnéia, os neonatos com cardiopatias congênitas podem recusar precocemente o seio materno pelo fato de não conseguirem coordenar eficazmente o processo de sucção-de- 
glutição-respiração (1). Diante disso, o leite materno pode não suprir as necessidades calóricas e protéicas de crianças cardiopatas que têm o metabolismo elevado, e, assim, exige-se a complementação com fórmulas infantis hipercalóricas (6).

A alimentação complementar é encorajada às crianças nascidas com cardiopatias seguindo as mesmas recomendações para crianças normais. Deve se iniciar por volta do quarto ou sexto mês de vida, com outras fontes de nutrientes, por sucos de frutas, frutas, vegetais, carnes e cereais preparados em papa amassada sem retirar fibras e temperos. A alimentação deve ser rica em proteínas e ferro, fortificada com gordura e carboidratos, com restrição de sódio e excesso de líquidos (6).

$\mathrm{E}$, frequentemente, orienta-se aumentar a densidade calórica levemente mediante fórmulas concentradas com óleo de milho, ou triglicerídios de cadeia média. Desse modo, o lactente assimila mais calorias, apesar da ingestão de menor volume de alimentos (11).

Somando-se a isso, no estudo, foram relatadas intercorrências durante a alimentação das crianças, as quais estão destacadas por episódios de vômitos, dispnéia, recusa alimentar, refluxo gastroesofágico, sudorese na região frontal da cabeça, crises hipoxêmicas. De modo geral, a criança portadora de cardiopatias congênitas sente muitas dificuldades na alimentação oral, pois diferentes sinais e sintomas decorrentes do baixo débito cardíaco e da função miocárdia alterada são exacerbados no momento da alimentação. Lactentes com insuficiência cardíaca congestiva podem manifestar dificuldades durante a amamentação, com sudorese excessiva de pólo cefálico, palidez e irritabilidade (12).

As crises hipoxêmicas são desencadeadas por um decréscimo importante no conteúdo de oxigênio, cuja consequência é a inadequada oferta deste elemento para os tecidos na tentativa de satisfazer as demandas metabólicas (13). A sudorese decorre da vasoconstrição secundária ao aumento do tônus adre- nérgico, enquanto a dispnéia é usualmente sintoma precoce da insuficiência cardíaca instalada com o declínio progressivo da reserva cardíaca e ocorre aos pequenos esforços nas mamadas ou choro nos lactentes (14). O refluxo gastroesofágico nas cardiopatias torna-se mais evidente quando a criança apresenta lesão cardíaca significativa, resultando do retardo no esvaziamento gástrico, secundário à má absorção (3).

Em decorrência disso, para muitas crianças portadoras de cardiopatias congênitas e seus pais, o momento das refeições torna-se tenso. Nessa situação, a recusa alimentar constante pode ser severa, desencadeando deficiências nutricionais e alterações no comportamento alimentar infantil (5).

Nesse ensejo, o cuidado realizado pela enfermeira junto a crianças cronicamente doentes, em especial àquelas portadoras de cardiopatias congênitas, é permeado de conflitos, que vão desde dificuldades encontradas nas relações que se estabelecem com seus familiares, até o manejo da alimentação da criança. Com base nisso, as enfermeiras relatam que desempenham um cuidado muitas vezes limitado à realização de procedimentos técnicos, tais como incentivo à dieta oral ou aplicação de sondas para alimentação (15). Porém, cuidar de uma criança com doença crônica não é uma tarefa fácil e restrita a procedimentos técnicos, mas sim um desafio que os enfermeiros enfrentam, visto que devem orientar os pais da criança, os quais irão necessitar de apoio de diversas naturezas emocional e afetiva, bem como promover o intercâmbio social e repassar informações acerca da real condição da saúde da criança (16).

Geralmente, a equipe profissional se dedica especialmente à criança, esquecendo das dificuldades e necessidades da família, sendo que assistir a criança também implica em dar assistência a sua família (17). Nesse contexto, a enfermeira precisa adquirir habilidades técnicas para desenvolver inovações tecnológicas que envolvam a criança e seus familia- 
res, incluindo a utilização de estratégias lúdicas durante o momento das refeições e, também, na sua avaliação nutricional periódica.

Dessa forma, como medida de manutenção do padrão alimentar da criança, muitas mães no estudo mencionaram a utilização de estratégias, como o uso de colher para ingestão de líquidos, fracionamento dos alimentos em períodos regulares e o incentivo de diferentes opções de alimento no cardápio. Nesse ponto, o uso de tais métodos pode ter colaborado para que, segundo elas, as crianças tivessem o hábito alimentar preservado.

Em conformidade com tais achados, estudo aponta algumas estratégias de suporte nutricional que podem ser planejadas e implementadas por uma equipe multidisciplinar. Nesse caso, o enfermeiro e outros profissionais podem otimizar o atendimento às crianças com cardiopatias, na tentativa de interromper o processo de desnutrição e recuperar o crescimento infantil. São elas: introduzir lentamente com aumento gradativo e progressivo a alimentação, observando os limites da criança; desencorajar o uso de fórmulas enriquecidas com elevada concentração de sódio; preferir alimentos ricos em ferro e evitar grandes infusões de líquidos (6).

Assim, a reciprocidade no cuidado à criança, entre a equipe de saúde e a família, pode favorecer uma melhor identificação das necessidades da criança possibilitando o planejamento de um cuidado mais integral, holístico e humano. A partir disso, a troca de experiências entre os cuidadores pode possibilitar um melhor relacionamento entre os profissionais, a criança e sua família, minimizando, possivelmente, os problemas alimentares e o sofrimento da família com a doença e a hospitalização (17).

Por fim, conclui-se que foram avaliadas 132 crianças portadoras de cardiopatias congênitas com idade até 2 anos em duas instituições de saúde especializadas em doenças cardíacas. A maioria delas eram residentes e naturais de Fortaleza-CE, do sexo masculino, com mediana de até 8,5 meses de vida que estavam em acompanhamento ambulatorial ainda no período pré-operatório, sem comorbidades evidentes.

Em relação aos aspectos sociais e familiares abordados neste estudo, como observado, as famílias tinham baixo poder aquisitivo para manter o filho cardiopata e outros membros. Esse achado pode dificultar o acesso à condição nutricional adequada da criança e, por conseguinte, comprometer a intervenção cirúrgica e aumentar os riscos para complicações pós-operatórias.

Quanto às mães das crianças, estas viviam com companheiro fixo, apresentaram grau de escolaridade equivalente a mais de nove anos de estudo e se restringiam às atividades do lar. Neste sentido, conforme se acredita, o grau de escolaridade, a maior permanência no lar das mães e o apoio de um companheiro podem ser fatores protetores à saúde nutricional da criança portadora de cardiopatia congênita, pois o cuidado prestado requer mais tempo e habilidade dos pais, além do conhecimento básico sobre a doença.

No geral, a avaliação do estado alimentar das crianças portadoras de cardiopatias congênitas nesse estudo é condizente com a avaliação de outras crianças sem tal patologia. Por constituírem um grupo tão específico sob condição estável de saúde, amparado pela boa disponibilidade de cuidados dispensados pelos pais com relativo grau de escolaridade, essas crianças gozavam de adequada condição alimentar, mesmo emergindo de famílias com baixo poder aquisitivo. Ainda, como observado, houve poucos relatos de intercorrências durante as refeições, sendo o apetite considerado preservado segundo a percepção dos pais, apesar da elevada incidência de crianças nascidas cardiopatas com déficits nutricionais evidenciados pela literatura.

Conforme observado, a limitação do presente estudo se encontra no uso de referências restritas a livros e artigos relativamente antigos, tendo em vista que, especificamente, a temática em questão ainda é timidamente 
explorada em estudos atuais, principalmente por que essas crianças apresentam características físicas e fisiológicas peculiares e, muitas vezes, são classificadas como déficit nutricional quando comparadas aos valores padronizados para peso e estatura preconizados pela Organização Mundial de Saúde. Em face disso, esse estudo propõe a realização de pesquisas futuras com vistas à investigação do estado nutricional de cardiopatas entre si, bem como na análise alimentar quantitativa.

Com base no exposto, considera-se necessário maior envolvimento das enfermeiras na avaliação alimentar como parte específica do estado nutricional da criança cardiopata, especificamente na aplicação de estratégias que estimulem à alimentação, uma vez que o defeito cardíaco desencadeia grandes repercussões para a criança. Nesse contexto, a enfermeira pode atuar antecipadamente ao prevenir complicações, mediante orientações e ensino aos pais, ou mesmo intervir de forma terapêutica diante das respostas humanas apresentadas pelas crianças.

Agradecimentos: Ao CNPq pelo apoio financeiro.

\section{REFERÊNCIAS}

1. Rosamond W, Flegal K, Furie K, Go A, Greenlund K, Haase N et al. American Heart Association Statistical Update: Heart Disease and Stroke Statistics-2008 Update: A Report From the American Heart Association Statistics Committee and Stroke Statistics Subcommittee . Circulation. 2008; 117(4): e25-146.

2. Baltaxe E, Zarante I. Prevalence of congenital heart disease in 44,985 newborns in Colombia. Arch Cardiol Mex. 2006; 76(3): 263-8.

3. Steltzer M, Rudd N, Pick B. Nutrition care for newborns with congenital heart disease. Clin Perinatol. 2005; 32: 1017-30.

4. Mitchell M, Logan RW, Pollock JCS, Ja- mieson MPG. Nutritional status of children with congenital heart disease. $\mathrm{Br}$ Heart J. 1995; 73: 277-83.

5. Clemente C, Barnes J, Shinebourne E, Stein A. Are infant behavioural feeding difficulties associated with congenital heart disease? Child Care Health Dev. 2001; 27(1): 47-59.

6. Oba J. Terapia nutricional na criança com cardiopatia congênita. En: Ebaid, M. Cardiologia em pediatria: temas fundamentais. São Paulo: Rocca; 2000. p. 495512.

7. Ministério da Saúde (BR), Conselho Nacional de Saúde. Resolução No 196/96. Decreto No 93.933 de janeiro de 1987. Estabelece critérios sobre pesquisa envolvendo seres humanos. Bioética. 1996; 4(2 supl.): 15-25.

8. Nydegger A, Bines JE. Energy metabolism in infants with congenital heart disease. Nutrition. 2006; 22: 697-704.

9. Almeida CAN, Ricco RG, Del Ciampo LA. Avaliação do estado nutricional. En: Ricco RG, Del Ciampo LA, Almeida CAN. Puericultura: princípios e práticas: atenção integral à saúde. São Paulo: Atheneu; 2000. p. 57-89.

10. Damas BGB. A necessidade de informação e suporte aos pais de crianças portadoras de cardiopatias congênitas [Dissertação de Mestrado]. São Paulo: Escola de Enfermagem, Universidade de São Paulo; 2008.

11. Hockenberry MJ, Wilson D, Inkelstein M. WONG: Fundamentos de enfermagem pediátrica. 7ma. ed. Rio de Janeiro: Elsevier; 2006.

12. Kobinger MEB. A. Avaliação do sopro cardíaco na infância. J Pediatr. 2003; 79 (supl. 1): S87-S96.

13. Iwahashi ER, Cavalini JF. Crise hipoxêmica ou de cianose. En: Ebaid, M. Cardiologia em pediatria: temas fundamentais. São Paulo: Rocca; 2000. p. 213-21.

14. Gonçalves RC, Caramuru LH, Atik E. Insuficiência cardíaca. En: Ebaid, M. Car- 
diologia em pediatria: temas fundamentais. São Paulo: Rocca; 2000. p. 189-212.

15. Silva JB, Kirschbaum DIR, Oliveira I. Significado atribuído pelo enfermeiro ao cuidado prestado à criança doente crônica hospitalizada acompanhada de familiar. Rev Gaúcha Enferm. 2007; 28(2): 250-9.

16. Nóbrega VM, Collet N, Silva KL, Coutinho SED. Rede e apoio social das famílias de crianças em condição crônica. Rev. Eletr. Enf. [Internet]. 2010; 12(3): 43140. [citado 24 enero 2010]. Disponível em: http://www.fen.ufg.br/revista/v12/ n3/v12n3a03.htm.

17. Gomes GC, Erdmann AL. O cuidado compartilhado entre a família e a enfermagem à criança no hospital: uma perspectiva para a sua humanização. Rev Gaúcha Enferm. 2005; 26(1): 20-30. 